\title{
Exotics searches in jet final states with the ATLAS detector
}

\author{
Adam GIBSON, on behalf of the ATLAS Collaboration*广 \\ University of Toronto \\ E-mail: adam.gibsondcern.ch
}

\begin{abstract}
We summarize searches for physics beyond the Standard Model with events containing jets in the final state in pp collision data recorded with the ATLAS detector at the LHC. We consider up to $1.0 \mathrm{fb}^{-1}$ of data at $\sqrt{s}=7 \mathrm{TeV}$. The data are compared to Standard Model expectations with the goal of searching for new phenomena: heavy resonances, contact interactions and gravitationallymediated effects in large extra dimensions, microscopic quantum black holes. No evidence for physics beyond the Standard Model is found, so we present a variety of model-independent limits and limits on particular hypothetical new phenomena.
\end{abstract}

XXIst International Europhysics Conference on High Energy Physics

21-27 July 2011

Grenoble, Rhônes Alpes France

\footnotetext{
*Speaker.

$\dagger$ A. Gibson is now with the Department of Physics and Astronomy, Valparaiso University, 1610 Campus Drive East, Valparaiso, IN 46383, USA (e-mail: adam.gibson-even@valpo.edu). Formerly with the Department of Physics, University of Toronto, 60 Saint George Street, Toronto M5S 1A7, Ontario, Canada.
} 


\section{Introduction}

Jet signatures allow us to probe the highest energies directly accessible at the Large Hadron Collider. At high energies, QCD jet production is expected to be the dominant process at LHC, allowing us to probe QCD in a new regime and, especially, to search for evidence of new physics with cross sections potentially higher even than QCD jet production. This could be a new, massive, particle produced with a large cross section and decaying to jets, or the onset of a new phenomenon such as strong gravitational interactions in extra dimensions accessible above a threshold energy. We present a survey of ATLAS searches for new physics using jet final states including searches with monojet, dijet, and multi-jet signatures. In general, we begin with a model-independent search phase and then, if no evidence for new physics is found, we proceed with model-dependent exclusions of parameter space. We present results that analyze $\sqrt{s}=7 \mathrm{TeV}$ datasets collected with the ATLAS detector [1]. Depending on the analysis, the datasets range from $35 \mathrm{pb}^{-1}$ of data from 2010 running to $1 \mathrm{fb}^{-1}$ of data from 2011 running, the full dataset available at the time of EPS HEP 2011. We see no evidence for physics beyond the Standard Model, but set a variety of tight model-independent and model-dependent constraints on various new physics scenarios.

\section{Multi-Jet Final States: Search for Microscopic Black Holes}

In models with additional large, flat, extra dimensions, such as the paradigm due to ADD [2], the fundamental Planck scale $\mathrm{M}_{D}$ is smaller than the Planck scale observed in four dimensions, and gravity can become strong at the $\mathrm{TeV}$ scale. If this is true, microscopic black holes might be abundantly produced at the LHC. We assume a classical model of black hole production and a semi-classical decay model. We set the cross section to zero below a threshold mass $\left(\mathrm{M}_{t h}>\mathrm{M}_{D}\right)$ below which these assumptions are expected to break down. The black holes are expected to decay quickly, democratically, according to the number of Standard Model degrees of freedom available to the various possible final state particles. Compared to QCD, we expect an excess of events with many high energy jets, and a large amount of total transverse energy in the jets.

For our search region, we require one jet to have $\mathrm{E}_{T}>250 \mathrm{GeV}$ to guarantee good trigger efficiency, and then require a total of at least five jets with $\mathrm{p}_{T}>50 \mathrm{GeV}$ and $\sum p_{T}>2 \mathrm{TeV}$, where the sum is over the high- $\mathrm{p}_{T}$ jets. To estimate the background we normalize the low-multiplicity jet data to the high multiplicity using the 1.1 to $1.2 \mathrm{TeV}$ bin of $\sum p_{T}$, and extrapolate to high $\sum p_{T}$ using the shape of the low-multiplicity $\sum p_{T}$ distribution. This shape is, to good approximation, the same as for high-multiplicity QCD data. The high- and low-jet multiplicity distributions are shown in Figure 1. In a $35 \mathrm{pb}^{-1}$ data sample, we predict $3.7 \pm 1.0$ (stat) \pm 1.1 (syst) events in the signal region, and observe seven in data. We set 95\% CL limits on the cross section times acceptance of new physics contributions to this signal region at $0.29 \mathrm{pb}$. And, we set model-dependent limits that depend on $\mathrm{M}_{D}, \mathrm{M}_{t h}$, and $\mathrm{n}$ and are shown in Figure 1. Further details of this search can be found elsewhere [3].

\section{Dijet Mass and Angular Distributions}

A variety of models of new physics, including models with excited quarks and axigluons, predict resonant production of states decaying predominantly to two jets. Other models, such as 

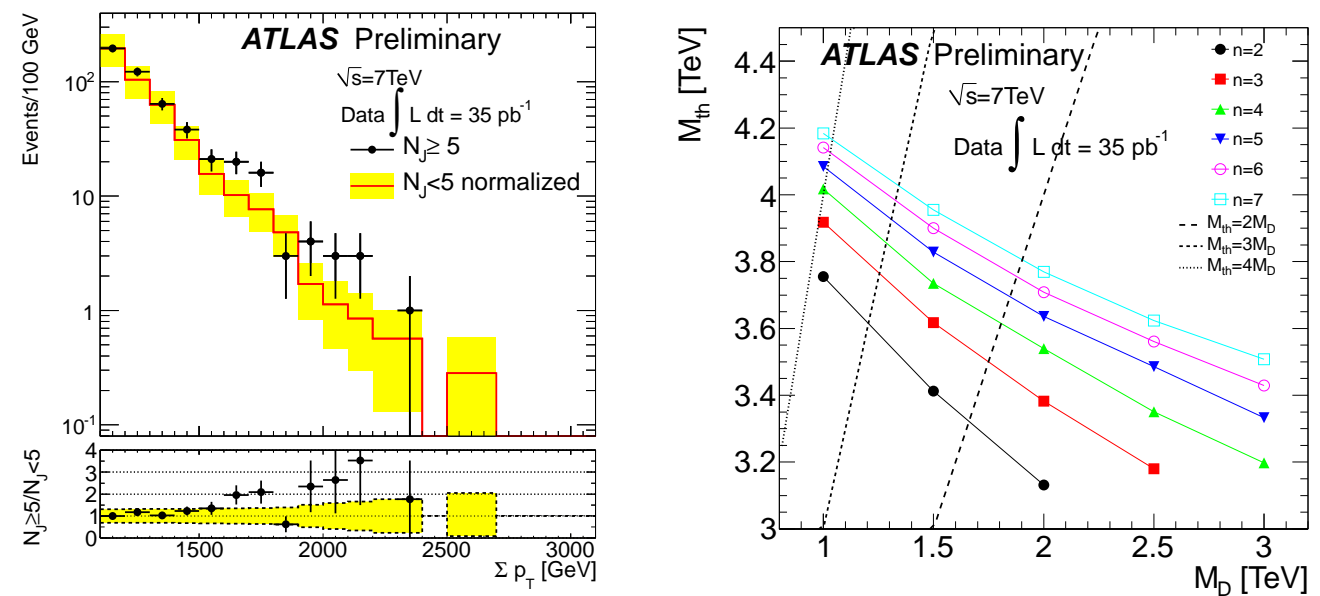

Figure 1: [Left] Scalar sum of jet transverse momentum $\left(\sum p_{T}\right)$ for observed events with less than five jets (histogram) and events with greater than or equal to five jets (points). The histogram is normalized to the number of events in the region $1.1<\sum p_{T}<1.2 \mathrm{TeV}$. The yellow band represents the total uncertainty, including the statistical uncertainties and the systematic uncertainties added in quadrature. [Right] Contour plots of the observed and expected limits on the Planck scale $\left(\mathrm{M}_{D}\right)$ versus threshold mass $\left(\mathrm{M}_{t h}\right)$ : observed limits. The curves are labeled by the number of extra dimensions $n$. Lines of fixed ratio $\mathrm{M}_{t h} / \mathrm{M}_{D}=2,3$, and 4 are also shown.

quark contact interactions as a low energy manifestation of quark compositeness, predict an excess of central dijet events at high mass. We conduct a model-independent BumpHunt for excesses in the dijet mass spectrum on top of a smooth background function fit to data. Seeing no evidence for new physics in $0.81 \mathrm{fb}^{-1}$ of jet triggered data, we set limits on simplified Gaussian $\mathrm{m}_{j j}$ distributions, exclude excited quarks with masses less than $2.91 \mathrm{TeV}$, axigluons with masses less than $3.21 \mathrm{TeV}$, and color octet scalars with masses less than $1.91 \mathrm{TeV}$. The $\mathrm{m}_{j j}$ spectrum and an example of limits on cross section times acceptance are shown in Figure 2. Details of the search for dijet resonances can be found elsewhere [4]. Updated results with $1.0^{-1} \mathrm{fb}$ of data have recently been presented [7]. They show no evidence for new physics and extend the excluded regions to, for example, excited quark masses up to $2.99 \mathrm{TeV}$.

We also explicitly analyze the angular distribution of dijet events by considering normalized distributions of $\chi=e^{\left|y_{1}-y_{2}\right|}$ for leading (second-leading) $\mathrm{p}_{T}$ jet rapidities $y_{1}\left(y_{2}\right)$. At high masses, many models such as quark contact interactions and models predicting low multiplicity decays of Quantum Black Holes, predict an excess of central dijet events. The $\chi$ distributions in data are shown in Figure 3 along with background predictions from NLO QCD. We also consider the fraction of events, as a function of $\mathrm{m}_{j j}$, where the dijets have proximate rapidities, $\mathrm{F}_{\chi}\left(\mathrm{m}_{j j}\right)$. This variable has excellent sensitivity to resonant as well as non-resonant excesses of central jets, and is shown in Figure 3 . Using $36 \mathrm{pb}^{-1}$ of data we set limits on the compositeness scale, $\Lambda$, for contact interactions at $6.7 \mathrm{TeV}$ and on the Planck mass, $\mathrm{M}_{D}$ in models of low multiplicity Quantum Black Hole decay with six extra dimensions at $3.67 \mathrm{TeV}$. Details of the angular dijet searches can be found elsewhere [5]. A recent review discusses dijet searches at the LHC and elsewhere [6]. 

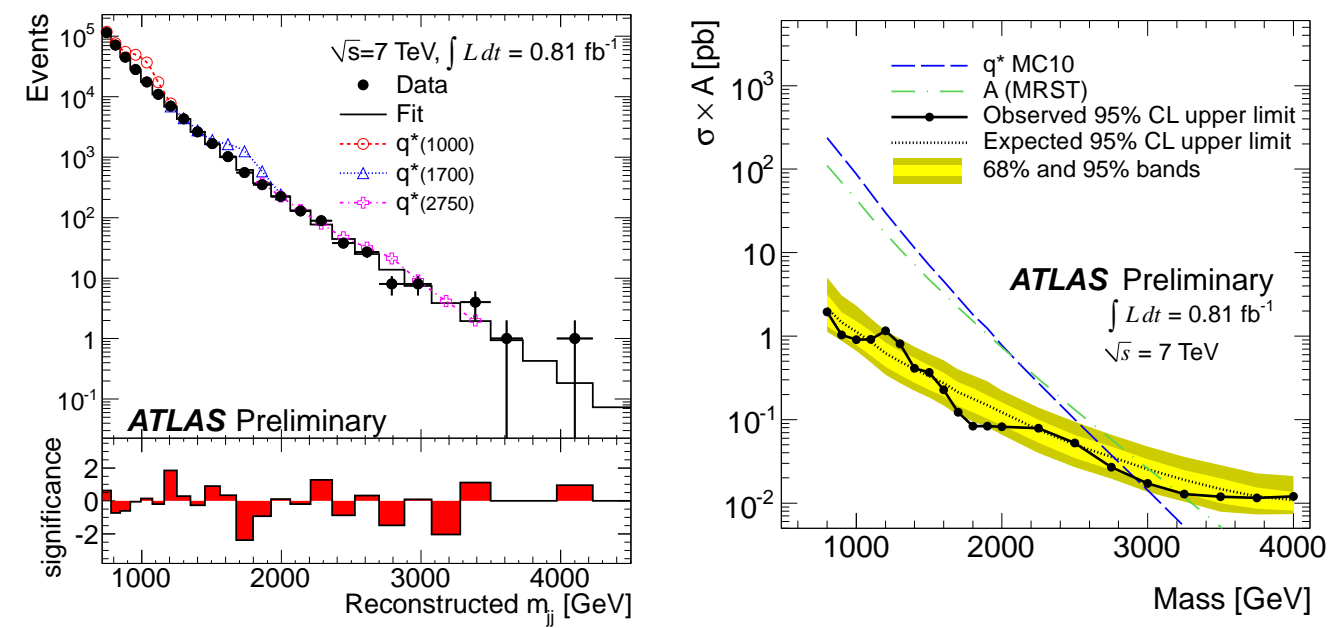

Figure 2: [Left] The observed (Data) dijet mass distribution (filled points) fitted using a binned QCD background (Fit) distribution described by the smooth functional form (histogram). The bin-by-bin significance of the data-background difference is shown in the lower panel. For illustration, excited quark mass templates have been stacked on the background distribution. [Right] Limit setting using cross section times acceptance theory curves for excited quarks (blue dashed) and axigluons (green dot-dashed). Black filled circles are the 95\% CL upper limit from data for $\sigma \times \mathrm{A}$ as a function of $\mathrm{m}_{j j}$. The black dotted curve shows the 95\% CL upper limit expected from Monte Carlo and the light and dark yellow shaded bands represent the $68 \%$ and $95 \%$ contours of the expected limit, respectively. For a given new physics model, the observed (expected) limit occurs at the crossing of its $\sigma \times$ A curve with the observed (expected) $95 \%$ CL upper limit curve.
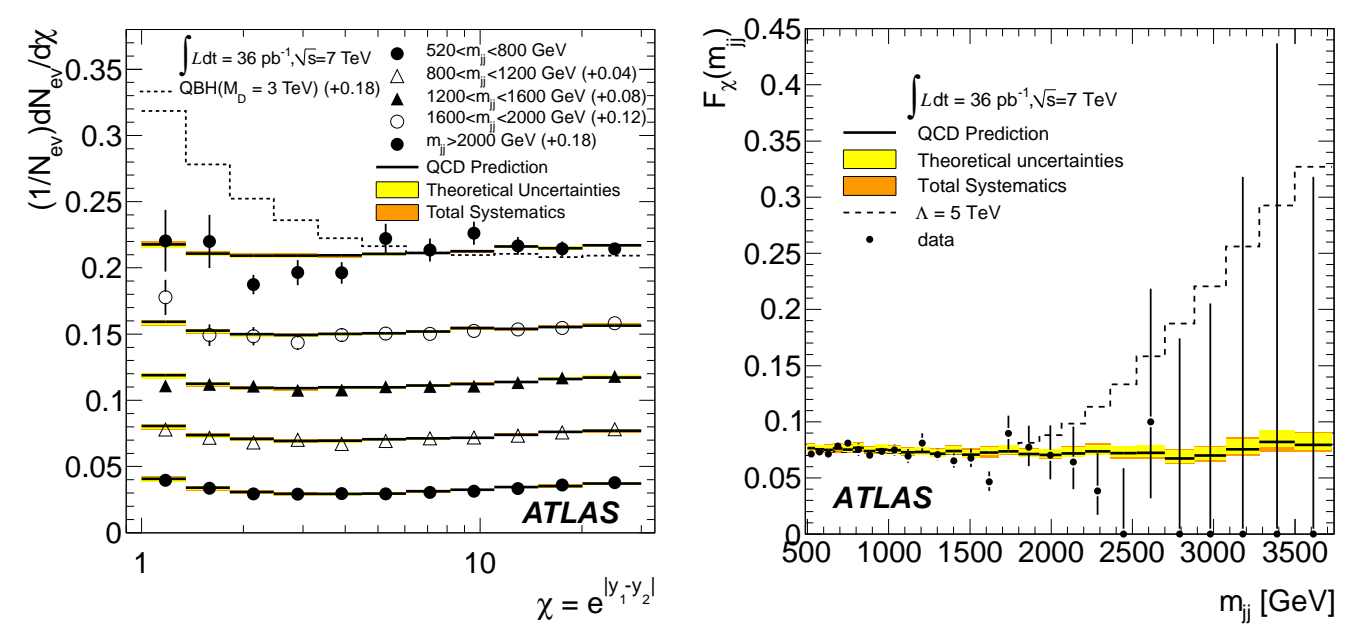

Figure 3: [Left] The $\chi$ distributions for $520<m_{j j}<800 \mathrm{GeV}, 800<m_{j j}<1200 \mathrm{GeV}, 1200<m_{j j}<1600$ $\mathrm{GeV}, 1600<m_{j j}<2000 \mathrm{GeV}$, and $m_{j j}>2000 \mathrm{GeV}$. Shown are the QCD predictions with systematic uncertainties (narrow bands), and data points with statistical uncertainties. The dashed line is the prediction for a QBH signal for $M_{D}=3 \mathrm{TeV}$ and $\mathrm{n}=6$ in the highest mass bin. The distributions and QCD predictions have been offset by the amount shown in the legend to aid in visually comparing the shapes in each mass bin. [Right] The $\mathrm{F}_{\chi}\left(\mathrm{m}_{j j}\right)$ function versus $\mathrm{m}_{j j}$. We show the QCD prediction with systematic uncertainties (band), and data points (black points) with statistical uncertainties. The expected signal from QCD plus a quark contact interaction with $\Lambda=5.0 \mathrm{TeV}$ is also shown. 

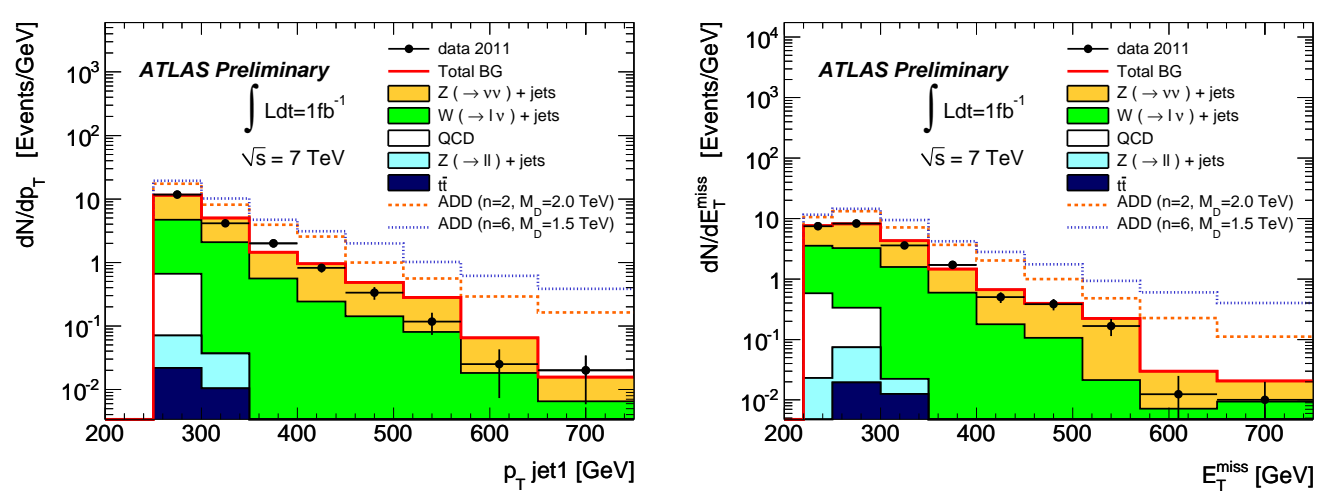

Figure 4: [Left] Measured leading jet $\mathrm{p}_{T}$ distribution (black dots) in the HighPt region compared to the predictions for SM backgrounds (histograms). [Right] Measured missing transverse momentum distribution (black dots) in the HighPt region compared to the predictions for SM backgrounds (histograms). In both plots, for illustrative purposes, only statistical uncertainties are shown and the impact of two different ADD scenarios is included.

\section{Search with a Monojet (plus Missing Transverse Energy) Signature}

Another possible consequence of large extra dimensions is an excess of events with a monojet signature, where, for example, a jet is produced balancing a graviton and the graviton disappears into an extra dimension. We would observe a single high- $\mathrm{p}_{T}$ jet and large missing $\mathrm{E}_{T}$. For the 1 $\mathrm{fb}^{-1}$ analysis we use a "HighP ${ }_{T}$ " selection requiring a leading-jet $\mathrm{p}_{T}$ above $250 \mathrm{GeV}$ and missing $\mathrm{E}_{T}$ above $220 \mathrm{GeV}$. We reject events with an electron or muon candidate to reduce the Electroweak background, and we reject events with a second-leading-jet above $60 \mathrm{GeV}$, a third-leading-jet above $30 \mathrm{GeV}$, or where the $\phi$ of the second-leading jet is within 0.5 radians of the missing $\mathrm{E}_{T}$ to reduce the QCD background. The shape of the dominant Electroweak background is taken from Monte Carlo and the normalization from data. The background and data distributions are shown in Figure 9 . The single largest background is from Z's decaying to neutrinos, estimated at 610 events. The total background is $1010 \pm 37$ (stat) \pm 65 (syst) events compared to 965 events in the signal region. This translates to model-independent limits on cross section times acceptance at $0.11 \mathrm{pb}$, and limits on $\mathrm{M}_{D}$ for $\mathrm{ADD}$ extra dimensions of, for example, $3.16 \mathrm{TeV}$ for two extra dimensions and $1.99 \mathrm{TeV}$ for six extra dimensions. Details of the monojet search can be found elsewhere [8].

\section{References}

[1] The ATLAS Collaboration, JINST 3, S08003 (2008)

[2] N. Arkani-Hamed, S. Dimopoulos and G. R. Dvali, Phys. Lett. B 429 (1998) 263 [arXiv:hep-ph/9803315].

[3] The ATLAS Collaboration, ATLAS-CONF-2011-068. https://atlas.web.cern.ch/Atlas/GROUPS/PHYSICS/CONFNOTES/ATLAS-CONF-2011-068/

[4] The ATLAS Collaboration, ATLAS-CONF-2011-095. https://atlas.web.cern.ch/Atlas/GROUPS/PHYSICS/CONFNOTES/ATLAS-CONF-2011-095/

[5] The ATLAS Collaboration, New J. Phys. 13 (2011) 053044 [arXiv:1103.3864 [hep-ex]]. 
[6] R. M. Harris, K. Kousouris, Submitted to: Int.J.Mod.Phys.A. [arXiv:1110.5302 [hep-ex]].

[7] The ATLAS Collaboration, arXiv:1108.6311 [hep-ex]. Submitted to Physics Letters B.

[8] The ATLAS Collaboration, ATLAS-CONF-2011-096. https://atlas.web.cern.ch/Atlas/GROUPS/PHYSICS/CONFNOTES/ATLAS-CONF-2011-095/ 